\title{
ALMOST RIEMANN INTEGRABLE FUNCTIONS
}

\author{
MOUSTAFA DAMLAKHI
}

\begin{abstract}
An arbitrary function $f$ on a bounded interval $[a, b]$ is termed an almost $R$-integrable function if there exists a Riemann integrable function $g$ such that $f=g$ a.e. In this note a characterization of the class of almost $R$-integrable functions is obtained.
\end{abstract}

\section{Introduction.}

A bounded function $f(x)$ defined on a bounded interval $[a, b]$ is Riemann integrable if and only if $f(x)$ is continuous a.e. But a function which is a.e. equal to a Riemann integrable function on $[a, b]$ need not be Riemann integrable as the characteristic function of the set of rational points in the interval shows. In this note, we characterize the class of functions on $[a, b]$ which are a.e. equal to Riemann integrable functions.

Here, the measure and the integral (unless otherwise specified) are taken in the sense of Lebesgue.

\section{A.lmost $R$-integrable functions:}

Definition 1. An arbitrary function defined on a bounded interval $[a, b]$ is said to be almost $R$-integrable if there exists a bounded function $g(x)$ on $[a, b]$ integrable in the sense of Riemann such that $f(x)=g(x)$ a.e.

Example of an unbounded function in $[0,1]$ that is almost $R$-integrable is

$$
f(x)= \begin{cases}n & \text { if } x=\frac{1}{n} \\ 1 & \text { otherwise }\end{cases}
$$

Note, however,

$$
g(x): \begin{cases}\frac{1}{\sqrt{x}} & \text { if } x \in(0,1) \\ 0 & \text { if } x=0\end{cases}
$$

Received January 18, 1994 revised April 8, 1994.

1991 Mathematics Subject Classification 26A42

Key words and phrases. Almost integrability. 
is Riemann integrable in the extended sense but not almost $R$-integrable

Proposition 2. An almost $R$-integrable function on $[a, b]$ is integrable (in the sense of Lebesgue) but not conversely.

Proof. It is obvious that $f$ is integrable if it is almost $R$-integrable.

Now, we exhibit an integrable function that is not almost $R$-integrable.

Let $A$ be a nowhere dense perfect set of measure $\frac{1}{2}$ in $[0,1]$.

If $f(x)$ is the characteristic function of $A$, then $f$ is integrable, but not almost $R$-integrable.

For if $f=g$ a.e. where $g$ is Riemann integrable, we arrive at a contradiction since $g$ is continuous a.e. while $f$ is not continuous at every one of the points of $A$ which is of measure $\frac{1}{2}$.

\section{The class $\mathcal{E}$ of functions:}

Let $\mathcal{E}$ denote the class of functions $f$ on $[a, b]$ such that $f$ is the limit a.e. of an increasing sequence $\left(\varphi_{n}\right)$ of step functions.

Theorem: A function $f$ defined on $[a, b]$ is almost $R$-integrable if and only if $f \in \mathcal{E} \cap-\mathcal{E}$.

Proof: Since every Riemann integrable function is in $\mathcal{E}$, it is clear that every almost $R$-integrable function $f \in \mathcal{E} \cap-\mathcal{E}$.

Conversely, let us suppose that $f \in \mathcal{E} \cap-\mathcal{E}$. Then $f^{+}$and $f^{-} \epsilon \mathcal{E} \cap-\mathcal{E}$; we'll therefore assume that $f$ is positive.

There exists then, an increasing sequence that $\left\{\varphi_{n}\right\}$ of step functions and another decreasing sequence $\left\{\psi_{n}\right\}$ of step functions satisfying the conditions: $\sup \varphi_{n}=f$ a.e. and inf $\psi_{n}=f$ a.e.

Remark that for some constants $A$ and $B$ and all $n$, we have $\int_{a}^{b} \varphi_{n}(x) d x \leq A$ and $\int_{a}^{b} \psi_{n}(x) d x \leq B$

By Lebesgue's monotone convergence theorem we also have

$$
\sup \int_{a}^{b} \varphi_{n} d x=\int_{a}^{b} f(x) d x=\inf \int_{a}^{b} \psi_{n} d x
$$

Consequently, given $\mathcal{E}>0$, we can choose $N$ so that

$$
\int_{a}^{b} \psi_{m} d x-\int_{a}^{b} \varphi_{n} d x<\epsilon \text { if } n, m \geq N .
$$


Now, by hypothesis $\varphi_{n} \leq \psi_{1}$ a.e. and since $\varphi_{n}$ and $\psi_{1}$ are step functions, the sequence $\left\{\varphi_{n}\right\}$ is bounded above.

Let $g(x)=\sup \varphi_{n}(x)$ on $[a, b]$. then $g(x)$ is a bounded function such that $g=f$ a.e. Now, it is enough to show that $g$ is Riemann integrable.

Let $\varphi_{N}(x)=\sum c_{k} X_{l_{k}}$

Take now a partition $P:\left(a=x_{0}<x_{1}<\cdots<x_{n}=b\right)$ of $[a, b]$ such that each $I_{k}$ is one of the subintervals of $P$. Then if $m_{p}$ is the lower Darboux sum of $g$ corresponding to the partition $P$, we have

$$
\int_{a}^{b} \varphi_{N} d x \leq m_{p} \leq \int_{\underline{a}}^{b} g(x) d x, \quad \text { where }
$$

$\int_{\underline{a}}^{b} g(x) d x$ denotes the lower integral of $g$ in the sense of Riemann.

In the same way, if inf $\psi_{n}(x)=h(x)$ then $h(x) \geq g(x)$ because $\psi_{m} \geq \varphi_{n}$ for any $n$ and $m$; also we can prove that $\int_{a}^{b} \psi_{N} d x \geq \int_{a}^{\bar{b}} h(x) d x \geq \int_{a}^{\bar{b}} g(x) d x$.

Consequently,

$$
\int_{a}^{\vec{b}} g(x) d x-\int_{\underline{a}}^{b} g(x) d x \leq \int_{a}^{b} \psi_{N} d x-\int_{a}^{b} \varphi_{N} d x<\epsilon .
$$

Since $\epsilon$ is arbitrary, we conclude that $g$ is Riemann integrable

This completes the proof of the theorem.

\section{Reference}

[1] Soo Bong Chae, Lebesgue integration, Marcel Dekker, Inc. 1980.

[2] F. Riesz and B. Sz. Nagy, Functional Analysis, Ungar Publishing Co., Sixth printing, 1972. 